\title{
Functional characterization of an IBD risk gene
}

C1ORF106 has an important role in regulating intestinal permeability, according to new research. The work also shows that an IBD-associated polymorphism in the gene encoding C1ORF106 disrupts protein stability and thus impairs gut barrier function.

Aberrant intestinal permeability has been associated with risk of IBD, and previous studies have suggested that host genetics might have a role in mediating gut barrier abnormalities. Genome-wide association studies have identified the ${ }^{\star} 333 \mathrm{~F}$ variant of C1ORF106 (also known as INAVA, encoding innate immunity activator protein) as associated with increased risk of IBD. The unclear function of C1ORF106 in intestinal homeostasis or IBD prompted Kara Lassen and colleagues to determine the role of the protein in physiological or pathophysiological settings.

"We first used proteomics to identify proteins that interact with C1ORF106," explains Lassen. This work identified cytohesin 1 and cytohesin 2 as major C1ORF106interacting proteins. Focusing on cytohesin 1 (a guanine exchange factor that controls the activation of the ARF6 GTPase), subsequent experiments using C1orf106-1 mice and cell lines showed that C1ORF106 regulates cytohesin 1 levels by inducing its ubiquitylationdependent degradation. Loss of C1ORF106 led to higher levels of cytohesin 1, thereby increasing ARF6 activity.

ARF6 has an important role in regulating cell-surface levels of adherens junction proteins, particularly E-cadherin. In cultured C1ORF106 ${ }^{-/-}$intestinal epithelial cells, surface levels of E-cadherin were reduced; colonic tissue samples from C1orf106-/- mice also showed reduced cell-surface E-cadherin levels. Importantly, there were no localization differences in other epithelial cell junction proteins (including occludin, ZO1, claudin 1 or claudin 2), and no differences in levels of their mRNA or protein.

Next, Lassen and colleagues assessed the influence of C1ORF106 on barrier function. Loss of C1ORF106 in mice increased intestinal permeability to small solutes and markedly increased bacterial translocation when the animals were challenged with Citrobacter rodentium.

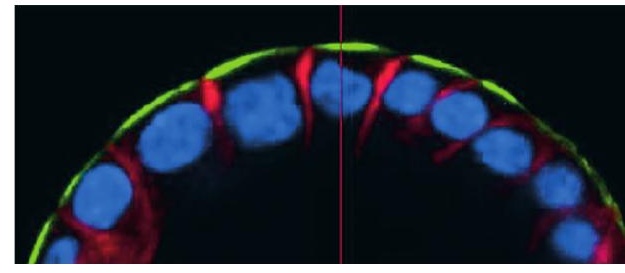

A C1orf106 $6^{+/+}$colonic organoid. Courtesy of V. Mohanan.

Finally, the authors explored the effect of the ${ }^{\star 333 F ~ C 1 O R F 106}$ variant. Degradation assays demonstrated that the ${ }^{\star} 333 \mathrm{~F}$ variant had a shorter half-life and was less stable than the wild-type protein. Downstream of C1ORF106, the $\star 333 \mathrm{~F}$ variant was associated with decreased gut barrier integrity owing to disrupted E-cadherin function. "The next steps are to understand how we can use the information we have learned about this pathway to develop therapies aimed at strengthening the intestinal barrier," concludes Lassen.

Hugh Thomas

ORIGINAL ARTICLE Mohanan, V.et al. C1orf106 is a colitis risk gene that regulates stability of epithelial adherens junctions. Science https://doi.org/10.1126/science.aan0814 (2018) 